\title{
Cord Malaria Infection, Complement Activation, Oxidative Stress, Gestational Age, and Birth Weight, Characterized by High Plasmodium falciparum Prevalence in Bamenda, Cameroon
}

\author{
Oumar Mahamat ${ }^{D}$, Kidio Gisele Ndum, Sumo Laurentine, and Ntonifor Ngum Helen \\ Laboratory of Biological Sciences, Faculty of Science, The University of Bamenda, Bamenda, Cameroon \\ Correspondence should be addressed to Oumar Mahamat; oumahamat@yahoo.com
}

Received 20 March 2020; Revised 25 July 2020; Accepted 29 July 2020; Published 11 August 2020

Academic Editor: Aditya Prasad Dash

Copyright (c) 2020 Oumar Mahamat et al. This is an open access article distributed under the Creative Commons Attribution License, which permits unrestricted use, distribution, and reproduction in any medium, provided the original work is properly cited.

\begin{abstract}
Background. It is unknown whether the presence of Plasmodium falciparum malaria parasites in umbilical cord blood denotes activation of complement and oxidative stress to affect the duration of pregnancy and birth weight. Methods. In a cross-sectional study conducted from January to April 2019 in Bamenda, Cameroon, cord blood samples were collected from 300 women at delivery. Parasitaemia was determined microscopically. Babies' weight and age of gestation were recorded. Plasma levels of complement and oxidative stress were measured by specific tests. Results. Cord blood malaria prevalence was $21.33 \%$. Babies with an infected cord showed a low birth weight and gestation age than those with uninfected cords. More babies with infected cords had LBW (6.25\%) compared to the counterparts (5.50\%). The levels of parasitaemia and the babies' weight showed a weak positive correlation. The prevalence of preterm and postterm birth was $4.33 \%$ and $24.33 \%$ respectively, with a weak negative correlation between the age of gestation and the umbilical cord parasitaemia. There was correlation between cord parasitaemia and levels of complement haemolytic activity titter $\left(\mathrm{CH}_{50}\right)$ and specific classical pathway activity (CPA) in cord blood. $\mathrm{CH}_{50}$ and $\mathrm{CPA}$ levels, however, were significantly higher in infected cord blood samples, compared with uninfected cord blood samples. $\mathrm{CH}_{50} \mathrm{showed} \mathrm{a}$ negative correlation with the birth weight and gestational age in infected cord blood samples. The levels of total oxidative stress (TOS) and total antioxidant defense were significantly lower in infected cord blood than uninfected. TOS displayed a positive correlation with the density of parasitaemia and a weak negative correlation with the birth weight and gestational age in infected cord blood. Conclusion. Cord blood infection lowers the complement haemolytic titter, oxygen radicals and total antioxidant defense in neonates. This lowering of complement haemolytic titter and oxygen radical compounds in umbilical cord malaria are associated with low birth weight and preterm birth.
\end{abstract}

\section{Introduction}

Malaria is a disease caused by parasites belonging to the Plasmodium genus. The parasites are transmitted to humans through the bite of infected female Anopheles mosquitoes. Malaria remains the first endemic parasitic disease in the world. In 2017, it was responsible for about 219 million clinical cases of sickness and 435,000 deaths worldwide. About $92 \%$ of cases and $93 \%$ deaths were reported in sub-Saharan Africa (SSA) [1]. In Cameroon, in 2013 for example, $28.7 \%$ of the population in health facilities consulted for malaria, and this scourge was responsible for $22 \%$ deaths in general population and 29 to $28 \%$ in neonates $[2,3]$.

Mother-to-child transmission constitutes in malaria as one of the major problems compromising the foetal development. It represents a risk factor for preterm birth, foetal growth restriction, and low birth weight [4-6]. Low birth weight that results constitutes a cause of foetal and neonatal morbidity and one of the important determinants of infant healthy growth and development $[7,8]$. Mother malariarelated low birth weight is estimated to up to $20 \%$ in an endemic area, with a relatively high risk of preterm delivery $[4,9]$. 
However, the mechanism that leads to low birth weight or preterm delivery in vertical transmission of malaria was not clearly understood. Diverse factors are suggested to be responsible. Anaemia related to immune activities are thought to be associated with malaria-related low birth weight $[5,6,10]$. The complement system, an essential part of innate immunity, may play a role. It is known to be important in initiation of acquired immune responses in malaria-infected individuals [11]. During malaria infection, the level of C5a has been described to be high [11, 12]. The increased level of C5a may induce the release of reactive oxygen species which indirectly contribute to foetal growth restriction.

In the past decades, numerous researches showed a wide-spread involvement of the complement system in a number of placental malaria processes [13]. Thus, measurement of the complement system and oxidative stress levels in cord blood serum may be an important tool to assess the disease impact and the health state of neonates in malaria. Identifying the cause of low birth weight and preterm delivery in malaria has been the focus of many studies, and several mechanisms have been proposed over the past decades. However, there is a lack of consensus concerning validation, standardization, and reproducibility. Here, we aim to assess the prevalence of umbilical cord malaria and discuss the contribution of the complement system through oxidative stress in malaria-related low birth weight and preterm delivery.

\section{Materials and Methods}

2.1. Study Area. The study took place at the Regional Hospital of Bamenda. Bamenda is the capital of the Northwest Region, Cameroon. Bamenda lies between latitude $5^{\circ} 94^{\prime} \mathrm{N}$ and $5^{\circ} 98^{\prime} \mathrm{N}$ and longitudes $10^{\circ} 15^{\prime} \mathrm{E}$ and $10^{\circ} 18^{\prime} \mathrm{E}$ [14]. It sits along the Cameroon Volcanic Line with two distinct relief features: a High Lava Plateau of about 1,400 m, and the Lower Plateau, with an average altitude of $1100 \mathrm{~m}$ above sea level both separated by a vast escarpment. It has a tropical climate with two seasons, a long rainy season of eight months (March to October) and a short dry season of four months. Malaria transmission in Northwest Cameroon is perennial but seasonal and peaks during the rainy season. Plasmodium falciparum is responsible for more than $90 \%$ of malaria infections [2].

2.2. Study Population. The study participants were pregnant women who voluntarily consented to permit collection of blood from their cord during labour and satisfied the inclusion criteria. Inclusion criteria were confirmation of the active phase of labour and asymptomatic, nonfebrile parturient and singleton pregnancy without any known congenital anomaly. Exclusion from the study were subjects that refused to participate in the study; presence of any medical illness such as diabetes mellitus, chronic renal disease, haemoglobinopathies, HIV/AIDs, filarial infection, chronic hypertension, and severe pre-eclampsia/eclampsia; and presence of multiple gestation, intrauterine foetal death, and obvious foetal anomalies. There were 300 women recruited having met the inclusion criteria.

2.3. Clinical Procedures. Women delivering at the health facility, after giving informed consent, were asked a standard series of questions focused on sociodemographic characteristics, history of fever, antimalarial drug use, clinical malaria during pregnancy, and the use of antimalarial chemoprophylaxis. During labour, five millilitres of blood were drawn by venepuncture from the clamped cord immediately after delivery. Neonates and placentas were weighted with an electronic digital scale ( \pm 10 grams). The Dubowitz scoring system was used to estimate the gestational age, using findings from physical and neurologic examinations. Scoring by the APGAR index was performed at delivery but was not recorded in this study. Blood samples were separated into two: $1 \mathrm{ml}$ into ethylenediamine tetra acetic acid (EDTA) tubes and the remaining in tubes without EDTA. Serum was collected from the tubes without EDTA.

2.4. Parasitological Analysis. Thick smears of cord blood were prepared from each sample, stained with Giemsa stain, and examined for the presence of malarial parasites using routine microscopy to determine parasitaemia. For positive slides, $t(\mathrm{~F} 1)[15,16]$. Parasite density was graded as low (parasites $<1,000 / \mu \mathrm{L})$, moderate $(1,000-4,999 / \mu \mathrm{L})$, and high $(>5,000 / \mu \mathrm{L})$ as reported by Owa et al. [9]. Parasite densities (parasite/ $\mu$ l of whole blood) were then calculated. All slides were double-read. If the ratio of densities from the first two readings was greater than 1.5 or less than 0.67 or if fewer than 30 parasites were counted with a difference of more than 10 parasites between the two readings, the slide was evaluated a third time. The geometric mean of the parasite density of the two most concordant results of the three readings was taken as the final result.

Parasites/ $\mu$ blood

$$
=\frac{\text { Number of parasites cunted } x 8000 \mathrm{WBC} / \mu \mathrm{l}}{\text { Number of WBC counted }} .
$$

2.5. Determination of Haemolytic Complement Titters. Complement titters were based on the classical haemolytic assay (CH50) for lysis of antibody-sensitized sheep E. $[17,18]$ In detail, $2 \times 10^{8} \mathrm{E} / \mathrm{ml}$ were opsonized with rabbit anti-sheep haemolysin (Sigma-Aldrich, MO) by adding an equal volume of a 1:160 dilution of haemolysin to the $\mathrm{E}$ suspension, and the mixture was, then, incubated at $37^{\circ} \mathrm{C}$ in a shaking water bath for 30 minutes. The sensitized $\mathrm{E}$ were, then, centrifuged at 3,000 rpm for five minutes and washed three times with gelatine veronal buffer (GVB) (SigmaAldrich Inc, supplemented with $10 \mathrm{mM}$ EDTA (GVBE). Sensitized E were stored in GVBE supplemented with $2.5 \%$ glucose, $0.03 \% \mathrm{NaN}_{3}$ at $4{ }^{\circ} \mathrm{C}$ for not more than two weeks. For use, sensitized erythrocytes were washed with GVB containing $\mathrm{Mg}^{2+}$ and $\mathrm{Ca}^{2+}\left(\mathrm{GVB}^{++}\right)$. 
Cord blood sera (in duplicates) were, then, serially diluted in a 96 micro-well plate (NUNC) in $\mathrm{GVB}^{++}$to give a final dilution of $1 / 320$. To each serum dilution, $25 \mu \mathrm{l}$ of $5 \%$ sensitized $\mathrm{E}$ was added followed by incubation at $37^{\circ} \mathrm{C}$ for $60 \mathrm{~min}$. The reaction was stopped by adding $100 \mu \mathrm{l}$ of GVBE to all wells. Control wells included spontaneous lysis of opsonized E without serum and $100 \%$ lysis well in which complete lysis was achieved by adding $1 \%$ triton X. The plate was centrifuged at $3000 \mathrm{rpm}$ in a plate centrifuge at $4^{\circ} \mathrm{C}$ for $5 \mathrm{~min}$ to pellet unlysed cells. $100 \mu \mathrm{l}$ of supernatant was, then, transferred to a flat-bottomed plate, and the absorbance readings of released haemoglobin were read at $415 \mathrm{~nm}$ using a microplate reader. The degree of lysis $(\mathrm{Y})$ was determined from the formula (2) $[18,19]$. The serum titter that causes $50 \%$ haemolysis of sensitized sheep E was determined using SPSS software.

$$
\mathrm{Y}=\frac{\text { OD sample }- \text { OD spontaneous lysis control }}{\text { OD triton lysis - OD spontaneous lysis control }} \text {. }
$$

2.6. Determination of Functional Complement Activity in the Pathways. The functional complement activity in classical and alternative pathways was evaluated using ELISA kits (Sigma) supplied precoated with specific activators for the classical pathway (CP) and alternative pathway (AP). Serum samples were, then, diluted into buffers containing specific blockers of each pathway. Samples were diluted at $1: 100$ and $1: 18$ for the CP and AP, respectively. Positive and negative controls with known activities were used for quality control of the assay and for calculation of the functional activities.

In detail, $100 \mu \mathrm{l}$ of each diluted sample and control were added in duplicate to the wells of the respective pathwayspecific plates and incubated for $70 \mathrm{~min}$ at $37^{\circ} \mathrm{C}$. Wells were, then, aspirated and washed thrice with wash buffer. Then, $100 \mu \mathrm{l}$ of conjugate containing alkaline phosphatase-labelled antibodies to C5b-9 was added to each well and incubated for $30 \mathrm{~min}$ at $25^{\circ} \mathrm{C}$. Wells were again washed thrice, and $100 \mu \mathrm{l}$ of substrate $\left(\left(3,3^{\prime}, 5,5^{\prime}\right.\right.$-tetramethylbenzidine (TMB) $)$ was added, and the samples were incubated for $30 \mathrm{~min}$ at $25^{\circ} \mathrm{C}$. Absorbance was, then, immediately read at $405 \mathrm{~nm}$. Functional activity for the respective pathways was, then, calculated using the OD values of the positive and negative controls.

2.7. Estimation of Plasma Total Oxidative Stress. Total oxidative stress (TOS) was measured by quantifying alkoxyl $(\mathrm{RO} *)$ and peroxyl $(\mathrm{ROO} *)$ radicals as described by Piyashi et al. [19] One hundred microliter $(100 \mu \mathrm{l})$ of plasma diluted to 20 times in PBS was dissolved in $1 \mathrm{ml}$ of acetate buffer. $25 \mu \mathrm{l}$ of working chromogen ( $\mathrm{N}, \mathrm{N}$-dimethyl-p-phenylenediamine sulphate) solution was, then, added, and absorbance was taken at $505 \mathrm{~nm}$ by 6 minutes time-scan in a UV-VIS spectrophotometer. The absorbance values were obtained at 4 to 6 minutes for each sample against blank. The quantity of radical compounds was determined as proportional to the absorbance.

\subsection{Estimation of Plasma Total Antioxidant Defense.} Total antioxidant defense (TAD) was determined as reported by Piyashi et al. [19] based on the measurement of the reduction of the radical cation of the chromogen $(\mathrm{N}, \mathrm{N}$ dimethyl-p-phenylenediamine sulphate) by antioxidant compounds in the sample. In detail, $1 \mathrm{ml}$ of acetate buffer $(\mathrm{pH}=5.2)$ taken in a test tube, $25 \mu \mathrm{l}$ chromogen reagent that contains $\mathrm{N}, \mathrm{N}$-dimethyl-p-phenylenediamine sulphate, and 10 microliter $\mathrm{FeCl}_{3}$ solutions are added. Thereafter, $10 \mu \mathrm{l}$ of 20 times diluted plasma was added, and the absorbance was photometrically read at $505 \mathrm{~nm}$ at $37^{\circ} \mathrm{C}$. Total antioxidant defense of the sample was considered to be inversely correlated to the absorbance.

2.9. Definitions. Blood film results were considered to be positive if any asexual-stage parasites were identified and negative if no parasites were seen in 100 high-power fields. Prematurity was defined as a gestational age $<37$ weeks ( $\leq 245$ days), normal gestation age 251-280 days, and postterm $>280$ days as estimated by Dubowitz examination $[9,20]$ and low birth weight as a birth weight of $<2500 \mathrm{~g}$, normal body weight $2.5-4 \mathrm{~kg}$, and macrosomia $(>4 \mathrm{~kg})$ with a gestational age $\geq 37$ weeks [9].

2.10. Statistical Analysis. Data collected in the study questionnaire were verified and, then, double-entered. Data were analyzed using Graph Pad Prism software v. 5.20 and SPSS. The analysis included data from births of all enrolled participants. Continuous normally distributed data were described by their means and standard deviation. Proportions were compared using the Chi-square test, and normally distributed continuous variables were compared using the Student $t$-test. Statistical results were considered significant when the two-sided $P$ value was $\leq 0.05$.

2.11. Ethical Considerations. The study was discussed with health authorities and local leaders to obtain their assent. The study was reviewed and approved by the Faculty of Health Science ethical committee, University of Bamenda. Informed consent was obtained after the consent document was read to the women in the local language. For illiterate mothers, the informed consent discussion process was witnessed by an impartial individual.

\section{Results}

3.1. Characteristics of Recruited Women. Data were collected from three hundred women, with a majority (88\%) aged between 20 and 35 years. Amongst these women, 98.33\% were taking fansidar, and only $8(2.66 \%)$ had clinical malaria during pregnancy (Table 1).

3.2. Labour Outcomes. The mean value of the gestational age of women who participated in this study was 275.27 days $( \pm 16.33)$, with a majority delivering at the normal time with normal babies' birth weight. The mean (SD) placenta weight was $606.66 \mathrm{~g}( \pm 116.69)$, with a birth/placenta weight ratio of 5.38 (Table 2). 
TABLE 1: Overall prevalence of malaria with respect to demographic information.

\begin{tabular}{lcc}
\hline Characteristics & Frequency & Percent (\%) \\
\hline Age groups (yrs) & & \\
Age $<20$ years & 19 & 6.33 \\
Age between 20 and 35 years & 264 & 88 \\
Age $>35$ years & 17 & 5.66 \\
Chemoprophylaxis & 5 & \\
No & 295 & 1.66 \\
Yes & & 98.33 \\
Clinical malaria during pregnancy & 8 & \\
Yes & 292 & 2.66 \\
No &
\end{tabular}

3.3. Umbilical Cord Malaria Infections. Out of the 300 cords, there were $64(21.33 \%)$ positive blood films. The density of parasitaemia varied from low to high density. There were 18 (28.12\%), $36(56.25 \%)$, and $10(15.62 \%)$ of the cords that showed low, moderate, and chronic infection, respectively (Table 3).

3.4. Impact of Parasitaemia on Labour Outcomes. With respect to infection, birth weight and gestation age were significantly lower in women with umbilical cord malaria infection than in women who did not have placental malaria infection $(P \leq 0.05)$. Relative to the density of parasites, this showed that the gestational age had a positive correlation $(r=0.14)$, while the birth weight showed a negative correlation $(r=-0.16)$. However, these relationships were not significant $(P>0.05$; Table 4$)$.

3.5. Relationship between the Umbilical Cord Malaria Infections and Total Complement Haemolytic Activity and Specific Pathways Activation of Umbilical Cord Serum. Figure 1 shows the total haemolytic complement titter $\left(\mathrm{CH}_{50}\right)$ and specific complement classical and alternative activities of umbilical cord serum. The Levels of $\mathrm{CH}_{50}$ were significantly higher $(P<0.0001)$ in malaria-infected cords (INF), $\left(\mathrm{CH}_{50}=21.41 \pm 7.35 \mathrm{UEq} / \mathrm{mL}\right)$ than in noninfected cords (UNF), $\left(\mathrm{CH}_{50}=14.25 \pm 2.06 \mathrm{UEq} / \mathrm{mL}\right)$. In specific pathways, only the activities of the classical pathways showed a significantly higher difference at $P \leq 0.0001 \quad(\%$ activity $=60.91 \pm 40.06 \%)$ in INF than in UNF $(\%$ activity $=19.91 \pm 22.24 \%)$. In linear regression, the parasitaemia density showed a significant and negative correlation with total haemolytic complement ( $r=-0.47 ; P=0.005)$.

3.6. Relationship between Labour Outcomes and Complement Haemolytic Activity Titter of Cord Blood Serum with Respect to the Umbilical Cord Parasitaemia. In umbilical cord malaria-positive women, $\mathrm{CH}_{50}$ was observed to correlate positively with the gestational age and the baby's birth weight, while in umbilical cord malaria-negative women, it was positively and negatively correlated with the gestational age and the baby's birth weight, respectively. Comparative analysis showed that, in relation to the gestational age, either the delivery was premature, normal,
TABLE 2: Distribution of the studied population according to the gestational age and birth weight.

\begin{tabular}{lcc}
\hline Characteristics & Frequency & Percent (\%) \\
\hline Gestation age or days from ovulation (days) & \\
Preterm ( $\leq 245$ days) & 13 & 4.33 \\
NGA (251-280 days) & 214 & 71.33 \\
Postterm ( $>280$ days) & 73 & 24.33 \\
Mean \pm SD & $275.27 \pm 16.33$ & \\
Birth weight $(\mathrm{kg})$ & & \\
LBW $(<2.5 \mathrm{~kg})$ & 263 & 5.66 \\
NBW $(2.5-4 \mathrm{~kg})$ & 20 & 87.66 \\
Macrosomia $(>4 \mathrm{~kg})$ & $3.21 \pm 0.58$ & 6.66 \\
Mean birth weight \pm SD & & \\
Placental weight $(g)$ & $606.66 \pm 116.69$ & \\
Mean placenta weight $\pm \mathrm{SD}$ & $5.38 \pm 0.83$ & \\
Bw/Pw ratio &
\end{tabular}

Bw, body weight. Pw, placenta weight. LBW, low birth weight. NBW, normal birth weight. NGA, normal gestation age.

TABle 3: Prevalence of umbilical cord blood malaria and the level of parasitaemia density/ $\mu$ in Bamenda Regional Hospital, Cameroon.

\begin{tabular}{|c|c|c|c|}
\hline & Mean \pm SD & Frequency & Percent $(\%)$ \\
\hline \multicolumn{4}{|c|}{ Prevalence of malaria parasitaemia in the cord } \\
\hline Not infected & - & 236 & 78.66 \\
\hline Infected & $3435.5 \pm 4366.72$ & 64 & 21.33 \\
\hline \multicolumn{4}{|c|}{ Classification of malaria parasitaemia density/ $\mu l$ of cord } \\
\hline Low & $696.88 \pm 188.69$ & 18 & 28.12 \\
\hline Moderate & $2448 \pm 1214.08$ & 36 & 56.25 \\
\hline High & $11920 \pm 5365.90$ & 10 & 15.62 \\
\hline
\end{tabular}

Low (parasites $<1000 / \mu \mathrm{L}$ ); moderate (parasites between $1000-4999 \mu \mathrm{L}$ ); high (parasites $>5000 / \mu \mathrm{L})$.

or postterm, and the $\mathrm{CH}_{50}$ was high in women with infected cords than in counterpart controls. In contrast, the specific classical pathway activity was low in umbilical cords without parasites than in infected cords for any category of delivery (Table 5).

3.7. Impact of Umbilical Cord Malaria Infections on Cord Blood Serum Oxidative Stress and Total Antioxidant Defence. Determination of the total oxidative stress (TOS) and total antioxidant defence (TAD) was based on the optical density, whose values are known to be positively and negatively correlated with total oxidative stress and total antioxidant defence. Here, in relation with the TOS of the cord blood serum, data showed that the optical density value was significantly $(P<0.0001)$ lower in case of INF compared to the corresponding UNF (healthy control group). When evaluating the TAD, optical density was significantly $(P<0.0001)$ high in case of INF as compared to UNF cords (Figure 2). Parasitaemia density was found to be significantly associated with total oxidative stress $(r=0.89, P<0.0001)$ and total antioxidant defence $(r=0.59 ; P=0.0003)$, with a positive correlation.

3.8. Role of Total Oxidative Stress and Total Oxidative Defense in the Labour Outcomes with Respect to the Umbilical Cord Density of Parasitaemia. In case of preterm delivery, as well 
TABLE 4: Labour outcomes in relation to the presence of malaria parasites in umbilical cords.

\begin{tabular}{lccc}
\hline Labour outcomes & \multicolumn{2}{c}{$\begin{array}{c}\text { Mean values of factors associated with infection } \\
(-) \text { cords }\end{array}$} \\
\hline Gestation age (days) & $(+)$ cords & $279.77 \pm 3.21$ & $P$ value \\
Birth weight $(\mathrm{kg})$ & $3.188 \pm 0.59$ & $4.32 \pm 0.50$ & 0.048 \\
Placenta weight $(\mathrm{g})$ & $610.38 \pm 119.61$ & $592.97 \pm 104.98$ \\
${ }^{*}$ Percentage of low birth weight (\%) & 6.25 & 5.50 & 0.041 \\
\end{tabular}

${ }^{*}$ Number of neonates with low birth weight.

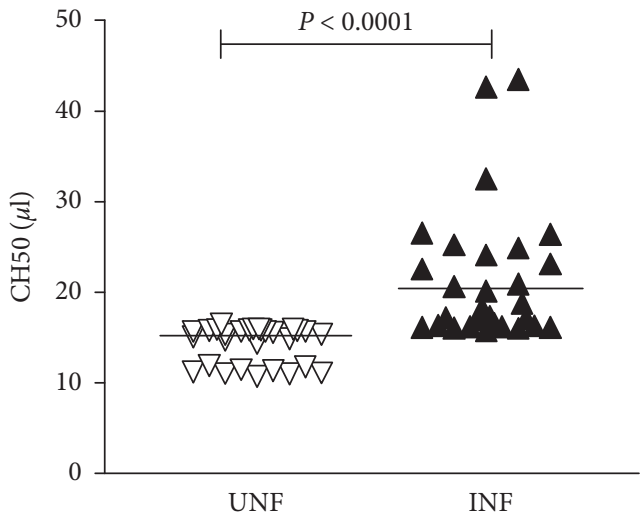

(a)

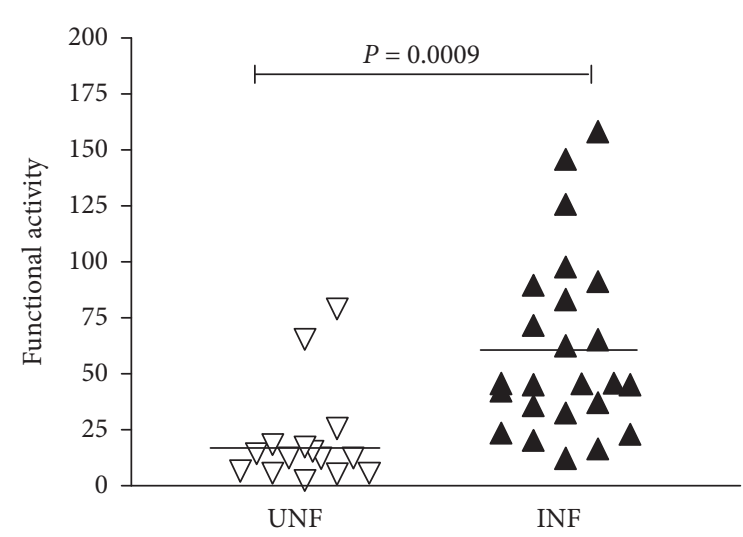

(b)

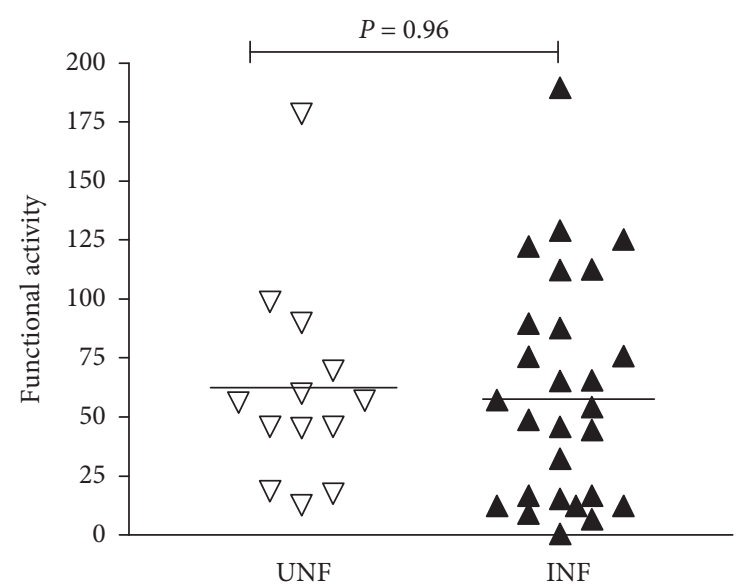

(c)

Figure 1: Haemolytic complement titter (CH50) and classical and alternative pathway activity of cord blood serum in relation to malaria parasites in cords. INF (malaria parasite-infected cords) and UNF (malaria parasite-uninfected cords). (a) CH50. (b) Classical pathway. (c) Alternative pathway.

as in normal and postterm deliveries, compared to UNF cords, the optical density recorded in determination of the total antioxidant defence and total oxidative stress was, respectively, high and low in infected cords. In infected cords, data showed that the gestational age was positively correlated $(r=0.11 ; P=0.53)$ with the TOS, while it was negatively correlated $(r=-0.88 ; P<0.0001)$ with TOS in uninfected cords (Table 6).

Independent on the birth weight value (low, normal, and high), a high optical density characterizing a low serum TAD was observed in uninfected cords compared to infected cords. However, in case of low birth weight, the optical density indicating the serum TOS was high in infected cords.
Furthermore, data showed that the birth weights correlated negatively $(r=-0.15 ; P=0.41)$ and positively $(r=0.58$; $P=0.0005)$ with TOS in the umbilical cord with malaria and without malaria, respectively (Table 6).

\section{Discussion}

This study aimed at assessing the prevalence of umbilical cord malaria and discussing the contribution of the complement system through oxidative stress in malaria-related low birth weight and preterm delivery at the regional hospital in Bamenda, Cameroon. The main outcomes of the present study were that the vertical transmission of malaria was 
TABLE 5: Complement haemolytic titer and classical pathway activity of cord blood serum following the delivery date and birth weight levels.

\begin{tabular}{|c|c|c|c|c|}
\hline \multirow{2}{*}{ Delivery outcomes } & \multicolumn{2}{|c|}{ Umbilical cord malaria-negative women } & \multicolumn{2}{|c|}{ Umbilical cord malaria-positive women } \\
\hline & $\mathrm{CH}_{50}$ & $\mathrm{CPA}$ & $\mathrm{CH}_{50}$ & $\mathrm{CPA}$ \\
\hline \multicolumn{5}{|l|}{ Age of gestation } \\
\hline Less than 245 days & $14.6 \pm 2.09^{\mathrm{a} \#}$ & $64.5 \pm 32.0^{\mathrm{a} \#}$ & $23.6 \pm 1.8^{\mathrm{b \#}}$ & $10.9 \pm 2.9^{\mathrm{b} \#}$ \\
\hline 245 days to 280 days & $14.7 \pm 1.82^{\mathrm{a} \#}$ & $71.1 \pm 31.8^{\mathrm{a} \#}$ & $20.1 \pm 4.6^{\mathrm{b} \#}$ & $31.5 \pm 26.2^{\mathrm{b} \#}$ \\
\hline 280 days and above & $13.6 \pm 2.34^{\mathrm{a} \#}$ & $75.6 \pm 37.2^{\mathrm{a} \#}$ & $23.7 \pm 10.6^{\mathrm{b \#}}$ & $23.2 \pm 21.5^{\mathrm{bt}}$ \\
\hline$r(P$ value $)$ & \multicolumn{2}{|c|}{$0.83(P<0.0001)$} & \multicolumn{2}{|c|}{$0.14(0.42)$} \\
\hline \multicolumn{5}{|l|}{ Baby's weight at birth } \\
\hline Less than $2.5 \mathrm{~kg}$ & $13.1 \pm 2.5^{\mathrm{a} \#}$ & $33.9 \pm 21.4^{\mathrm{a} \#}$ & $24.4 \pm 2.1^{\mathrm{b} \#}$ & $11.5 \pm 3.4^{\mathrm{b} \#}$ \\
\hline $2.50 \mathrm{~kg}$ to $4.0 \mathrm{~kg}$ & $14.6 \pm 1.8^{\mathrm{a} \#}$ & $68.4 \pm 29.4^{\text {a\# }}$ & $20.2 \pm 0.3^{\mathrm{b} \&}$ & $19.4 \pm 4.7^{\mathrm{b} \&}$ \\
\hline High than $4.0 \mathrm{~kg}$ & $12.5 \pm 2.8^{\mathrm{a \#}}$ & $95.5 \pm 48.5^{\mathrm{a} \#}$ & $20.7 \pm 1.1^{\mathrm{b} \& \mathrm{r}}$ & $17.9 \pm 0.8^{\mathrm{b} \&}$ \\
\hline$r(P$ value $)$ & \multicolumn{2}{|c|}{$-0.65(P<0.0001)$} & \multicolumn{2}{|c|}{$0.008(0.96)$} \\
\hline
\end{tabular}

$\mathrm{CH}_{50}$ (complement haemolytic titter), $\mathrm{CPA}$ (classical pathway activation), $r$ (Person's correlation coefficient with $\mathrm{CH}_{50}$ ). Symbol letters indicate the difference following delivery outcomes, while letters show the difference related to the umbilical cord malaria.

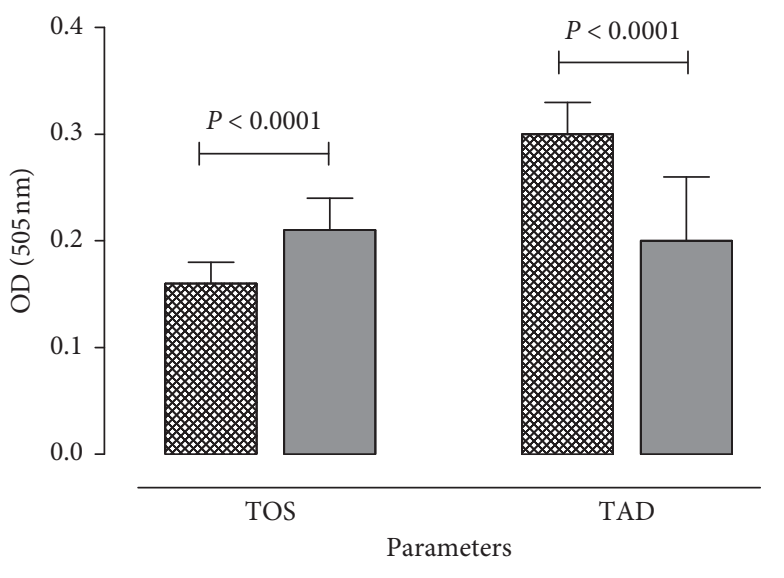

Infected patients

$\square$ Uninfected patients

FIGURE 2: Optical density related to total oxidative stress and total antioxidant defence of cord blood plasma in relation to cord malaria infection. Chart represents Mean \pm SD of optical density $(n=2)$.

$21.33 \%$. This prevalence was despite the intermittent preventive treatment (IPT) based on fansidar. Thus, cord malaria should be given greater attention in malaria control strategies that might include changes of actual drugs, fansidar. Clinical malaria of a mother during pregnancy has been shown not to be associated with cord infection. Thus, asymptomatic malaria of pregnant women should be given greater attention in the prevention of vertical transmission of malaria.

In the current study, the frequency of cords with high density of parasitaemia was higher than the frequency of cords of women with clinical malaria during pregnancy. This demonstrated that vertical transmission of malaria is not a matter of having a clinical malaria in the mother during pregnancy (or peripheral parasitaemia $>5000$ parasites $/ \mu \mathrm{L}$ ) as previously reported [21]. Maternal malaria during pregnancy, even in case of low parasitaemia, can result in vertical transmission.

The findings of this study showed a high percentage of preterm delivery and low birth weight in women with cord malaria. Likewise, previous studies demonstrated the impact of the vertical transmission on the baby's body weight at birth and the duration of pregnancy. Preterm birth, foetal growth restriction, and low birth weight were associated with congenital malaria [22-24].

In the present study, the cord blood plasma haemolytic titter of the complement system $\left(\mathrm{CH}_{50}\right)$ was significantly higher in the infected cord than their counterpart control uninfected. The presence of malaria parasite in the cord might activate the complement system, and subsequently, it results in low $\mathrm{CH}_{50}$. In a specific pathway functional screen test, data showed that the cord blood plasma of infected cords had high specific classical pathways activities than those of noninfected cords. This suggests that the classical pathway activation, a strict antibody-dependent pathway, might not be affected as the alternative pathway which was low in infected cords. The complement system is a fundamental element of the normal host defence against malaria infection [10]. A generally low value of this immunity component suggests that cord malaria might result in the disruption of immune responses in the neonates.

Furthermore, plasma levels of cord total oxidative stress and total antioxidant defence were significantly lower in malaria-infected than in noninfected women. This finding raises the important question of whether the presence of parasites in cord blood results in oxidative stress in neonates. Oxygen radicals have been demonstrated to be important for the clearance of parasites $[25,26]$. Therefore, low oxygen radicals as observed in this study suggest that the high prevalence of umbilical cord malaria may demonstrate inactivity of immune cells, notably phagocytes, the main source of oxygen radicals. Low oxygen radicals of infected cords might also be attributed to low complement components serving in opsonization processes and known to induce the oxidative stress status.

The complement system has been linked to many disease symptoms and complications, including poor pregnancy outcomes $[10,27,28]$. In the current study, serum blood $\mathrm{CH}_{50}$ was observed to correlate positively with the gestational age and the baby's birth weight in a malaria-infected cord, while in noninfected cords, it was negatively correlated with the baby's birth weight. Placental malaria was also suggested to be the main determinant of low birth weight 
TABLE 6: Total oxidative stress and total oxidative defence of cord blood serum following the delivery date and birth weight levels.

\begin{tabular}{|c|c|c|c|c|}
\hline & \multicolumn{2}{|c|}{ Umbilical cord malaria-negative women } & \multicolumn{2}{|c|}{ Umbilical cord malaria-positive women } \\
\hline & TAD & TOS & TAD & TOS \\
\hline \multicolumn{5}{|l|}{ Age of gestation } \\
\hline Less than 245 days & $0.28 \pm 0.02^{\mathrm{a} \#}$ & $0.14 \pm 0.03^{\mathrm{a} \#}$ & $0.13 \pm 0.01^{\mathrm{b} \#}$ & $0.19 \pm 0.01^{\mathrm{b} \#}$ \\
\hline 245 days to 280 days & $0.29 \pm 0.03^{\mathrm{a} \&}$ & $0.16 \pm 0.06^{\mathrm{a} \&}$ & $0.18 \pm 0.05^{\mathrm{b} \&}$ & $0.21 \pm 0.03^{\mathrm{b} \&}$ \\
\hline 280 days and above & $0.30 \pm 0.04^{\mathrm{a} \&}$ & $0.18 \pm 0.02^{\mathrm{a} \&}$ & $0.18 \pm 0.04^{\mathrm{b} \& \mathrm{r}}$ & $0.21 \pm 0.03^{\mathrm{b} \& \mathrm{r}}$ \\
\hline$r(P$ value $)$ & \multicolumn{2}{|c|}{$-0.84(<0.0001)$} & \multicolumn{2}{|c|}{$0.11(0.53)$} \\
\hline \multicolumn{5}{|l|}{ Baby weight at birth } \\
\hline Less than $2.5 \mathrm{~kg}$ & $0.31 \pm 0.03^{\mathrm{a} \#}$ & $0.12 \pm 0.02^{\mathrm{a} \#}$ & $0.15 \pm 0.03^{\mathrm{b} \#}$ & $0.20 \pm 0.01^{\mathrm{b} \#}$ \\
\hline $2.50 \mathrm{~kg}$ to $4.0 \mathrm{~kg}$ & $0.27 \pm 0.03^{\mathrm{a} \&}$ & $0.16 \pm 0.11^{\mathrm{a} \&}$ & $0.19 \pm 0.05^{\mathrm{b} \&}$ & $0.15 \pm 0.03^{\mathrm{a} \&}$ \\
\hline High than $4.0 \mathrm{~kg}$ & $0.26 \pm 0.04^{\mathrm{a} \&}$ & $0.18 \pm 0.01^{\mathrm{a} \&}$ & $0.18 \pm 0.05^{\mathrm{b} \&}$ & $0.16 \pm 0.01^{\mathrm{b} \& \mathrm{r}}$ \\
\hline$r(P$ value $)$ & \multicolumn{2}{|c|}{$0.58(0.0005)$} & \multicolumn{2}{|c|}{$-0.15(0.41)$} \\
\hline
\end{tabular}

TAD (total oxidative stress), TOS (total oxidative defence), $r$ (Person's correlation coefficient with TOS). Symbol letters indicate the difference following delivery outcomes, while letters show the difference related to the umbilical cord malaria.

[7, 29]. The positive correlation between $\mathrm{CH}_{50}$, birth weight, and gestational age, as observed in this study, suggests that the activation of complement resulting in low $\mathrm{CH}_{50}$ might be the cause of low birth weight. This is in line with recent observations that showed that soluble terminal complement complex levels were higher in placental plasma samples of newborns weighing less than $2700 \mathrm{~g}$ than in heavier newborns $[10,28]$ as the activation of the complement system that results in low $\mathrm{CH}_{50}$ can result in high soluble terminal complement complex levels.

Furthermore, the total oxidative stress was positively correlated with the gestational age, but negatively correlated with the birth weight. Low radical oxygen as observed in malaria-infected cords in consequence of a low complement system subsequent to infection might induce preterm birth or low birth.

\section{Conclusions}

Findings of this study revealed that cord malaria remains prevalent in Bamenda, Cameroon, with a high rate of asymptomatic carriage of malaria parasites. The level of cord infection significantly influenced the birth weight and gestational age. In addition to the cord blood parasitaemia that constituted a source of preterm delivery and low birthweight, the low complement activities, low total oxidative stress, and antioxidant defence of cord blood during vertical transmission might reveal an additional interesting cause of low birth weight and preterm delivery in malaria endemic areas.

\section{Data Availability}

All relevant data generated and analyzed during this study are available within the article.

\section{Conflicts of Interest}

The authors declare that they have no conflicts of interest.

\section{Authors' Contributions}

OM conceived and designed the experiments, analyzed the data, and wrote the paper. KGN performed the experiments. SL contributed to write the paper. NHN edited the paper.

\section{Acknowledgments}

The authors are grateful to the mothers for taking part in the study. The authors thank project staff, including medical officers, nurses, and laboratory technicians of Regional Hospital of Bamenda, Cameroon. They also thank Mr. Hamet for his technical support and assistance in the immunological analysis.

\section{References}

[1] C. N. Mbohou, L. P. K. Foko, H. N. Nyabeyeu et al., "Malaria screening at the workplace in Cameroon," PLoS One, vol. 14, no. 12, Article ID e225219, 2019.

[2] MINSANTE, Plan stratégique national de lutte contre le paludisme au Cameroun 2014-2018, MINSANTE, Yaounde, Cameroon.

[3] Institut National de la Statistique (INS) et ICF, International. Enquête Démographique et de Santé et à Indicateurs Multiples $d u$ Cameroun 2011, INS et ICF International, Calverton, MD, USA, 2012.

[4] M. M. J. Al-Obaidi and A. W. Taylor-Robinson, "Regulation of expression of reactive oxygen intermediates during plasmodium infection to reduce immunopathology provides a possible antioxidant adjuvant to enhance anti-malarial drug therapy," International Journal of Clinical Microbiology, vol. 1, no. 1, pp. 8-15, 2017.

[5] L. S. Harriet, A. C. C. L. Lawford, K. Sailesh, G. L. Helen, and B. Samudragupta, "Establishing a conceptual framework of the impact of placental malaria on infant neurodevelopment," International Journal of Infectious Diseases, vol. 84, pp. 54-65, 2019.

[6] C. J. Uneke, "Impact of placental Plasmodium falciparium malaria on pregnancy and prenatal outcome in Sub-Saharan Africa: Introduction to placental malaria," Yale Journal of Biology and Medicine, vol. 80, no. 2, pp. 39-50, 2007.

[7] B. U. Oraneli, O. C. Okeke, and P. O. Ubachukwu, "Effect of placental malaria on birth weight of babies in Nnewi Anambra State, Nigeria," Journal of Vector Borne Diseases, vol. 50, no. 1, pp. 13-17, 2013.

[8] O. A. Sule-Odu, A. Ogunledun, and A. O. Olatunji, "Impact of asymptomatic malaria parasitaemia at parturition on perinatal outcome," Journal of Obstetrics and Gynaecology, vol. 22, no. 1, pp. 25-28, 2003.

[9] O. O. Owa, A. R. Eniowo, A. N. Adedosu, P. S. Ogunro, S. O. Faturoti, and A. A. Ogunro, "Prevalence of 
asymptomatic malaria parasitaemia during pregnancy and its effect on foetal birth weight," International Journal of Current Research and Review, vol. 9, no. 5, pp. 41-48, 2017.

[10] A. Alim, N. E. Bilal, A.-E. Abass, E. M. Elhassan, A. A. Mohmmed, and I. Adam, "Complement activation, placental malaria infection, and birth weight in areas characterized by unstable malaria transmission in central Sudan," Diagnostic Pathology, vol. 10, no. 1, p. 49, 2015.

[11] V. M. Luciana and P.-G. A. Carlos, "Placental malaria: from infection to malfunction," Cell Host \& Microbe, vol. 13, pp. 125-127, 2013.

[12] A. Muehlenbachs, M. Fried, J. Lachowitzer, T. K. Mutabingwa, and P. E. Duffy, "Genome-wide expression analysis of placental malaria reveals features of lymphoid neogenesis during chronic infection," The Journal of Immunology, vol. 179, no. 1, pp. 557-565, 2007.

[13] M. C. Behet, L. Kurtovic, G.-J. van Gemert et al., "The complement system contributes to functional antibody-mediated responses induced by immunization withPlasmodium falciparumMalaria sporozoites," Infection and Immunity, vol. 86, no. 7, 2018.

[14] C. Acho-Chi, "Human interphase and environmental instability: addressing the environmental problems of rapid urban growth," Environment and Urbanization, vol. 10, no. 2, pp. 161-174, 1998.

[15] E. R. Alves-Junior, L. T. Gomes, D. Ribatski-Silva et al., "Assumed white blood cell count of 8,000 cells/ $\mu$ l overestimates malaria parasite density in the Brazilian amazon," PLoS One, vol. 9, no. 4, Article ID e94193, 2014.

[16] A. B. Jalal, I. G. Gasim, H. K. Amani, M. E. Leana, and A. Ishag, "Malaria parasite density estimation using actual and assumed white blood cells count in children in eastern Sudan," Journal of Tropical Pediatrics, vol. 62, no. 2, pp. 171-175, 2016.

[17] D. K. Adam, V. B. Paul, D. S. Michael, J. D. David, A. L. Margaret et al., "Rituximab infusion promotes rapid complement depletion and acute CD20 loss in chronic lymphocytic leukemia," The Journal of Immunology, vol. 172, no. 5, pp. 3280-3288, 2004.

[18] K. N. Nancy, P. T. Ronald, N. M. Joseph, and N. W. John, "Complement consumption in children with Plasmodium falciparum malaria,” Malaria Journal, vol. 8, no. 1, p. 7, 2009.

[19] B. Piyashi, A. Lakshmisona, P. Prasenjit, D. Mousumi, D. Moumita et al., "Simple modified colorimetric methods for assay of total oxidative stress and antioxidant defense in plasma: study in diabetic patient," Archives of Medicine, vol. 7, no. 5, pp. 1-7, 2015.

[20] S. Sangeeta, B. Ritu, and B. Shivani, "Comparison of Dubowitz scoring versus Ballard scoring for assessment of fetal maturation of newly born infants setting," International Journal of Reproduction, Contraception, Obstetrics and Gynecology, vol. 6, no. 7, pp. 3096-3102, 2017.

[21] O. Alphonse, B. T. Alfred, D. Amidou, C. C. B. Edith, N. Issa et al., "Transplacental transmission of plasmodium falciparum in a highly malaria endemic area of Burkina Faso," Journal of Tropical Medicine, vol. 2012, Article ID 109705, 7 pages, 2012.

[22] S. Chawanpaiboon, J. P. Voge, A. B. Moller, P. Lumbiganon, M. Petzold, and D. Hogan, "Global, regional, and national estimates of levels of preterm birth in 2014: a systematic review and modelling analysis," Lancet Global Health, vol. 7, no. 1, pp. 37-46, 2019.

[23] A. C. Lee, N. Kozuki, S. Cousens, G. A. Stevens, H. Blencowe, and M. F. Silveira, "Estimates of burden and consequences of infants born small for gestational age in low and middle income countries with intergrowth-21(st) standard, analysis of CHERG datasets," BMJ, vol. 358, 2017.

[24] M. C. Hoffman, K. K. Rumer, A. Kramer, A. M. Lynch, and V. D. Winn, "Maternal and fetal alternative complement pathway activation in early severe preeclampsia," American Journal of Reproductive Immunology, vol. 71, no. 1, pp. 55-60, 2014.

[25] I. A. Clark and N. H. Hunt, "Evidence for reactive oxygen intermediates causing hemolysis and parasite death in malaria," Infection and Immunity, vol. 39, no. 1, pp. 1-6, 1983.

[26] B. Greve, L. G. Lehman, B. Lell, D. Luckner, R. Schmidt-Ott, and P. G. Kremsner, "High oxygen radical production is associated with fast parasite clearance in children withPlasmodium falciparumMalaria," The Journal of Infectious Diseases, vol. 179, no. 6, pp. 1584-1586, 1999.

[27] A. Khattab, P. G. Kremsner, and S. Meri, "Complement activation in primiparous women from a malaria endemic area is associated with reduced birthweight," Placenta, vol. 34, no. 2, pp. 162-167, 2013.

[28] A. H. Mohammed, M. M. Salih, E. M. Elhassan et al., "Submicroscopic Plasmodium falciparum malaria and low birth weight in an area of unstable malaria transmission in Central Sudan," Malaria Journal, vol. 12, no. 1, p. 172, 2013.

[29] C. Menendez, J. Ordi, M. R. Ismail et al., "The impact of placental malaria on gestational age and birth weight," The Journal of Infectious Diseases, vol. 181, no. 5, pp. 1740-1745, 2000. 